\title{
Conference Report: 10th International Congress of Radiation Research, Würzburg, Germany, August 27-September 1, 1995
}

\author{
Michael McKay, Peter van der Spek, Henk Roest, Dirk Bootsma * \\ Department of Cell Biology and Genetics, Medical Genetics Centre, Erasmus Unicersity, Rotterdam. The Netherlands
}

Received 31 January 1996; accepted 6 February 1996

\section{Introduction}

An International Congress of Radiation Research convenes every 4 years. The 10 th such congress was held in the German city of Würzburg, between August 27 and September 1, 1995.

This meeting was especially notable, for both historical and scientific reasons. It was aptly held in the city where 100 years previously, one of the seminal scientific discoveries was made by Wilhelm Conrad Röntgen, the discovery of X-rays. From the perspective of modern radiation biology research, a major new trend was evident in the work presented at this meeting: the increasing contribution and power of molecular genetic approaches in addressing fundamental questions in radiobiology, for example, the genetic events which determine the sensitivity of cells to ionizing radiation. It is clcar that grcat strides have been made in this area since the last International Congress of Radiation Research in Toronto, Canada, in 1991. This communication emphasises and attempts to present an overview of some of the ways in which molecular biology is contributing to, and influencing radiation research, as viewed through the 'snapshot' of this major radiation research congress. The report is selective, focussing on major

${ }^{*}$ Corresponding author. themes arising from the Congress lectures, symposia and workshops attended by the authors. We believe it reflects the current status of this field and illustrates some emerging trends.

We make no attempt to be comprehensive, as we could not hope to do justice to even the biology contributions among the 1281 scientific contributions at this congress, spanning the spectrum of radiation research. For this, the reader is referred to the published and upcoming congress proceedings. Because references are not included, the potential risks of misquoting dictate that only general concepts are presented. The authors accept responsibility for any errors of reporting or omisssion.

\section{DNA repair genes and molecular mechanisms of ionizing radiation-induced DNA damage repair}

A. Lehmann (University of Sussex, UK) discussed methods used to identify and isolate human DNA repair genes, with an emphasis on ionizing radiation repair genes. Successful methods adopted to date have included transfection complementation of genotoxin-sensitive mutants (e.g., ERCC genes, $X R C C 1$ ); approaches in which a functional in vitro assay has been used to purify a protein activity, followed by amino acid sequencing to allow synthe- 
sis of degenerate oligonucleotide primers, and identification of the corresponding cDNA sequence using PCR/library screening (e.g., XPC, PCNA); and by virtue of sequence homology to cloned DNA repair genes of lower species, typically yeast (e.g., HHR6A, $H H R 6 B, H H R 23 A, H H R 23 B)$. A further point was the influence of serendipity: the involvement of DNA repair genes in other cellular processes has been uncovered by others working in different areas of enquiry (e.g., $X P B$ and $X P D$ genes identified as part of the basal transcription machinery). Positional cloning has also been used in the search for ionizing radiation repair genes. For example, the XRCC5 gene was mapped to $2 \mathrm{q} 33-35$ by microcell-mediated human chromosome transfer followed by irradiation-fusion gene transfer (IFGT), prior to the finding that the gene encoding $\mathrm{Ku} 80$ was defective in the corresponding mammalian mutants. This speaker also noted the recently determined link between mammalian ionizing radiation sensitivity, DNA double-strand break rejoining, V(D)J recombination and possibly signal transduction brought about by the significant discovery that an abundant nuclear $\mathrm{Ku}$ antigen subunits and the DNA-dependent protein kinase catalytic subunit (DNA-PK ${ }_{c s}$ ) were defective in mammalian ionizing radiation-sensitive mutant cell lines (corresponding to human XRCC5 and -7 genes). A point of note in this regard was the recent cloning of a full-length (approx. $15 \mathrm{~kb}$ ) DNA-PK $\mathrm{cs}_{\mathrm{cDNA}}$ by $T$. Blunt in the speaker's laboratory. Transfection of this cDNA corrected DNA-PK activity, V(D)J recombination in a transient expression assay, and radiosensitivity of $\mathrm{V} 3$ Chinese hamster ovary $(\mathrm{CHO})$ mutant cells, although the radiosensitivity correction was not complete. One explanation offered for the latter finding was that the human and endogenous CHO proteins might not have the same physical configurations. The major recent achievement of the cloning of the gene responsible for ataxia-telangiectasia (AT), ATM, was also discussed. Among the increasing number of genes showing homology to ATM was the rad3 gene of Schizosaccharomyces pombe: a recent finding was that $\mathrm{A}$. Carr from his laboratory had identified a second human AT-related gene to which the rad3 gene was more closely homologous. A find point was the recent dramatic increase in cloning of human ionizing radiation repair genes, with the current list including $X R C C J-7$,
$A T M$, DNA-PK components, human homologs of RAD51,-52 and -54 genes of Saccharomyces cerevisiae, and DNA ligases I, III and IV.

The study of ultraviolet radiation repair genes has provided a paradigm for DNA repair. D. Bootsma (Erasmus University, The Netherlands) outlined work on the xeroderma pigmentosum (XP) complementation group $\mathrm{B}$ gene $(X P B / E R C C 3)$, which illustrated the fact that different mutations in the same gene can give rise to diverse clinical manifestations. Such conditions might give clues to the biological roles of these proteins, for example, the contribution of particular mutations to a predisposition to carcinogenesis. For a long time, only one individual with combined XP/Cockayne syndrome (CS) was known. This female manifested severe CS and XP clinical features and her fibroblasts had $<5 \%$ unscheduled DNA synthesis (UDS; a biochemical marker of NER function), and a frameshift at amino acid 742 (the protein is 782 amino acids in length) due to a splicing mutation defect of $X P B$. This individual succumbed to cancer at an early age. More recently, two brothers with XP/CS were identified with XPB mutations (phenylalanine to serine substitution at amino acid 99) and XP clinical and biochemical features approximately as severe as the first individual, but with very mild CS features. These individuals remain cancer-free at ages 38 and 39 . Very recently, the first known trichothiodystrophy (TTD) individual was found with a mutation (at amino acid 119) of the XPB protein. Like for CS, no increased incidence of cancer has been seen in TTD. This speaker also noted that a number of viable mouse models for NER have now been created. The list includes knockouts of $E R C C \%, X P A, X P C$ and $C S$ $B$, whilst experiments generating mice harbouring subtle mutations in other DNA repair genes (e.g., $X P B, X P D)$ are in an advanced stage in his laboratory.

The present status of cloning and mapping the $X R C C$ group of ionizing radiation repair genes was addressed by $\mathbf{L}$. Thompson (Lawrence Livermore National Laboratory, USA). A recent suggestion by this speaker was to adopt the ' $X R C C$ ' nomenclature for new human ionizing radiation repair genes which are isolated via complementation of rodent mutants. The major XRCC mutants, and the chromosomal localization of their complementing XRCC genes 
Table 1

Major mammalian ionizing radiation sensitive ' $X R C C^{\prime}$ mutants, grouped according to phenotype

\begin{tabular}{|c|c|c|c|}
\hline Phenotype & Mutant & Gene & $\begin{array}{l}\text { Chromosomal } \\
\text { localisation }\end{array}$ \\
\hline \multirow{2}{*}{$\begin{array}{l}\text { DNA dsb rejoining- } \\
\text { deficient }\end{array}$} & $\mathrm{XR}-1, \mathrm{M} 10$ & $X R C C 4$ & $5 q 13-q 14$ \\
\hline & $\begin{array}{l}\text { xrs5, XRV15B, sxi-3 } \\
\text { V3/SCID }\end{array}$ & $\begin{array}{l}X R C C 5 / K u 80 \\
X R C C 7 / S C I D / D N A-P K_{i}\end{array}$ & $\begin{array}{l}2 q 34-36 \\
8 p 11.1-q 11.1\end{array}$ \\
\hline \multirow[t]{2}{*}{$\begin{array}{l}\text { DNA single-strand break } \\
\text { rejoining-deficient }\end{array}$} & EM9, EM-C11 & $X R C C l$ & $19 q 13.2-q 13.3$ \\
\hline & irsSF & $X R C C 3$ & $14 q 32.3$ \\
\hline \multirow{2}{*}{$\begin{array}{l}\text { Strand break repair } \\
\text { "proficient" }\end{array}$} & irs 1 & $X R C C 2$ & $7 q 36$ \\
\hline & irs $2 / V-C 4$ & $X R C C 8$ & - \\
\hline
\end{tabular}

was summarised by this speaker, and is reproduced in Table 1. Attempted functional complementation of the irs SF mutant (XRCC3 gene) with an integrating cDNA library had yielded a cDNA which restored chromosomal instability to approximately wild-type levels; however, X-ray sensitivity correction was only around $40 \%$. The XRCC3 cDNA showed partial primary amino acid sequence homology to other genes involved in the ionizing radiation response ( RAD57 and RAD51 of S. cerevisiae and the human homolog of the latter gene, HsRAD51), including a nucleotide binding box. Data were also provided regarding a recently characterized mammalian mutant, UV40, representing the uncloned XRCC 9 gene based on a unique mutant phenotype. This mutant showed broad spectrum genotoxin sensitivity (including 2-fold to ionizing radiation; 4-fold to UV; 7-fold to ethylmethane sulphonate and 11 -fold to mitomycin C), a 4-fold increase in sister chromatid exchange, an increased chromosome aberration frequency, and, despite the UV sensitivity, wild-type UDS. Regarding attempts to generate an XRCCI knockout mouse, this speaker reported that experiments were far advanced, but homozygous null mutants were not yet obtained.

Experiments characterizing the scid phenotype were reported by C. Kirchgessner (Stanford University, USA). It was previously reported that the scid phenotype (e.g., ionizing radiation sensitivity and $\mathrm{V}(\mathrm{D}) \mathrm{J}$ recombination deficiency) was corrected by human chromosome 8 . Work from several groups, including that of this speaker, strongly suggested that DNA-PK $_{c s}$ and scid are the same gene; these data included complementation of the $\mathrm{V}(\mathrm{D}) \mathrm{J}$ recombination deficiency and radiosensitivity of scid cells (and cells from the V3 CHO mutant residing in the same complementation group) by YACS containing pericentric regions of human chromosome 8 , as well as restoration of DNA-PK activity by chromosome 8complemented scid/V3 cells. Mapping the scid locus was recently refined to $8 \mathrm{p} 11.1-\mathrm{q} 11.1$. Proof that the DNA-PK ${ }_{c}$ is the gene defective in scid mice will require identifying mutations in this gene in scid mice: recent cloning of the full-length cDNA for DNA-PK ${ }_{\mathrm{cs}}$ (see above) will facilitate this. A model for the role of DNA-PK in the processing of DNA dsbs was also presented, in which, after binding of the $\mathrm{Ku} 80$ and 70 subunits to DNA ends, the DNA$\mathrm{PK}_{\mathrm{cs}}$ is recruited, forming a scaffold bridging the DNA gap, with alteration of the local chromatin conformation. DNA-PK has been shown to phosphorylate many proteins in vitro, including numerous transcription factors, p53, topoisomerase II and replication protein $\mathrm{A}$, although the physiological downstream phosphorylation targets of DNA-PK in this major mammalian DNA dsb repair process are yet to be defined. That chromatin structure was important to the scid phenotype was further addressed: it was previously shown that homozygous scid mutant cells, defective in DNA dsb rejoining, were not concomitantly deficient in rejoining non-genomic (transfected plasmid) DNA, suggesting that an inability to pro- 
cess the DNA dsb in its native chromosomal conformation was an important factor in scid radiosensitivity. In investigating this, the sensitivity of scid cells to 3-aminobenzamide, an inhibitor of polyADPribosylation and therefore a modulator of chromatin structure, was examined: scid mutant cells were not found to be sensitive to this agent. In the discussion which followed this presentation, A. Lehmann made the comment that further support for the model of DNA-PK forming a scaffold structure at the site of DNA dsbs was that apart from just its large size, DNA-PK interacts with DNA on a wide front, with an approx. 100 nucleotide DNA footprint.

H. Roest (Erasmus University, The Netherlands) reported progress in the development of mouse models for homologs of the RAD6 gene of $S$. cerevisiae, a gene involved in postreplication repair. RAD6 mutants have a complex phenotype, with sensitivity to many genotoxins, including UV - and ionizingradiation. RAD6 is duplicated in mammals, and both genes are functionally homologous to the S.cerevisiae gene as shown by their ability to correct, to a large extent, the UV-sensitivity of a rad6 null-mutation. These mammalian genes are, however, not capable of correcting a sporulation defect in this yeast strain. The generation of knockout mice for the MR6B gene, one of the mouse homologs of $R \mathrm{AD} 6$, was presented. Fibroblasts isolated from homozygous mutant embryos did not show increased sensitivity to UV- or ionizing- radiation when compared with wild-type embryonal fibroblasts. An interesting feature was observed relating to the sporulation defect in some yeast rad6 mutants: homozygous mutant males showed a clear defect in spermatogenesis, whereas female germ cell development was normal.

The different categories of DNA dsb repair pathways were reviewed by M. Resnick (Research Triangle Park, USA), with an emphasis on recombinational DNA dsb rejoining in $S$. cerevisiae. A number of models exist for the latter process, with common features including strand invasion, exonuclease degradation of (one of) the involved DNA strands, and DNA synthesis. This speaker described a recent innovation developed in his laboratory: TAR (transformation associated recombination) cloning. This procedure was developed in response to the common problem that YACs are often rearranged. Early data with this approach showed a high efficiency of re- covery of non-chimeric human DNA, with implications for areas such as positional cloning by improved manipulation of large genomic fragments.

Xenopus (frog) oocytes have been used by $\mathbf{P}$. Pfeiffer (University of Koln, Germany) and colleagues to study DNA dsb rejoining after restriction enzyme-induced DNA dsbs in substrate plasmids. Such systems have a number of important advantages, including the fact that DNA can be injected directly into the nucleus (which can abrogate transfection artefacts previously described for mammalian cells), and because circular plasmid DNA is not replicated in oocytes, immediate- as well as endproducts of repair can be readily isolated for analysis. The systems are advocated as attractive for the study of non-homologous DNA end-joining, and experiments are in progress to fractionate the active extracts in an attempt to delineate the active components. An initial impression was that such systems were also promising for the study of ionizing radiation-induced DNA dsbs.

A number of mammalian genes have now been isolated by virtue of their homology with DNA repair genes of lower species. J.-M. Buerstedde (Basel Institute of Immunology, Switzerland) reviewed progress with the identification and characterization of eukaryotic homologs of the RAD52 cpistasis group of $S$. cerevisiae, genes involved in homologous DNA dsb recombinational repair. Genes structurally homologous to $R A D 51,-52$ and -54 have been cloned from higher species, indicating conservation of this repair pathway during eukaryotic evolution. In work from a number of laboratories, most of the genes from $S$. pombe, chicken, mouse and human corresponding to these three $R A D$ genes have now been identified. Consistent with their putative roles in recombination in mammals, the mammalian genes are expressed at relatively higher levels in lymphoid and gonadal tissues. With respect to the functional significance of the described structural homologies, to date, no complete functional complementation of yeast mutants has been reported with these genes, although some partial complementation has been observed (e.g., the corresponding human genes have partially complemented MMS sensitivity of RAD51 and RAD54 (see below) mutants and the mating-type switching defect in an $S$. pombe rad 22 mutant - the rad 22 gene of $S$. 
pombe being a structural homolog of $S$. cerevisiae RAD52). R. Kanaar (Erasmus University, The Netherlands) reported the cloning and analysis of a human gene structurally homologous to the RAD54 gene of $S$. cerevisiae. Degenerate oligonucleotide primers based on conserved regions of RAD54 and an $S$. pombe RAD54 equivalent, thp54, were used to PCR-amplify from a chicken cDNA library a fragment subsequently used to retrieve clones from a human cDNA library. The human gene had an open reading frame of $2241 \mathrm{bps}$, and contained conserved structural motifs placing it in the SNF2/SW12 family of putative DNA helicases. The mRNA of the mouse homolog of RAD54 was found to be elevated in organs of lymphoid and germ cell development. An exciting recent development was the report of the viability of embryonic stem cells with targeted homozygous deletions of the mouse RAD54 homolog, constructed in the same laboratory by J. Essers.

C. Lawrence (University of Rochester, USA) reported on work concerning the mutagenic potential of certain DNA lesions as a result of processing by repair systems which deal with such lesions during replication. Data were presented that the mutagenic effect of T-T cyclobutane dimers is mainly determined by the polymerase that is used in the repair process. There appears to be a DNA-polymerase-imposed preference to insert a purine, especially an adenine opposite the lesion ('A'-rule). The $S$. cerecisiae gene, REV3, encodes a non-essential, putative polymerase that is involved in translesion synthesis after UV radiation. REV3 was shown to interact with REV7 and, with both proteins present in a complex, is capable of bypassing T-T dimers in vitro. Computer database searches using REVI and REV 3 identified human expressed sequence tags with a homology of $31 \%$ and $45 \%$, respectively. At the same symposium, M. Goodman (University of Southern California, USA) reported on the lesion bypass ability of different polymerases and the effect of purified proteins like RecA, $U m u D$ and $U m u C$ in an in vitro lesion bypass system. This system consists of a 27 nucleotide single-strand template with a $\mathrm{T}-\mathrm{T}$ dimer and a 15-mer primer.

The cellular phenomenon of radioresistant DNA synthesis (RDS) has long been associated with the ionizing radiation sensitive ataxia telangiectasia and related disorders, and has also been observed in
'AT-like' mutant rodent cell lines. Using chromosome transfer techniques, M. Zdzienicka (Leiden University, The Netherlands), found that chromosome $4 q$ was able to complement the RDS defect in a $\mathrm{CHO}$ ionizing radiation-sensitive mutant. In this case, RDS was uncoupled from radiation sensitivity (the latter not being complemented), suggesting the mutant may be defective in a gene involved in mammalian DNA replication control.

\section{Heterogeneity of DNA repair}

Intragenomic heterogeneity of DNA repair in mammalian systems has been recognised for some time. Preferential repair of a number of different lesion types has been described for many active genes. The most extensively studied lesions are UV photoproducts, particularly cyclobutane pyrimidine dimers (CPDs). In separate presentations, L. Mullenders and A. van Zeeland (both of Leiden University, The Netherlands) addressed this topic. NER has been the paradigm for the elaboration of the concept of preferential repair. Two subtypes of NER have been described: RNA pol II $t$ ranscription-coupled NE $R$ (TCR; a relatively rapid process), and the slower NER restricted to the non-transcribed strands of active genes and the remainder of the genome, although for CPDs, the non-transcribed strand of active genes is still repaired, in general, faster than the remainder of the genome. The early methods, which powerfully defined the concept of heterogeneity of repair, had the relative disadvantage that repair could only be assessed by Southern analysis of large DNA fragments (of the order of $>20 \mathrm{~kb}$ ). More recent use of antibodies against specific lesions allows repair to be evaluated in much smaller fragments, using either Southern blot analysis or PCR. Further advantages of PCR for this use are that changes can be detected in situ, and at more physiologically relevant doses of the genotoxin in question.

In the case of XP complementation group $\mathrm{C}$, there is a defect in the repair of UV-photolesions (and some other lesions, such as crosslinks) in the genome overall, but no defect in transcription-coupled repair. The XP-C defect contrasts with a specific transcription-coupled repair defect in cells from CS patients, who are not cancer-prone: XP-C is therefore an 
important XP subgroup for carcinogenesis studies. These differences were also referred to by $D$. Bootsma in a previous session, who emphasised that it appears that defects in the process of repair in non-transcribed sequences could be responsible for carcinogenesis, and that mouse models of defects in these particular genes may be useful in studying this.

Many systcms, ranging from $E$. coli to mammals, have now been tested for the presence of DNA strand-specific and preferential repair of many different genotoxins (e.g., UV-photoproducts, alkylating and cross-linking agents and ionizing radiation). No cases of strand-specific repair have yet been reported in the absence of preferential repair of the lesion in question. Factors affecting strand-specificity and preferential repair include the genotoxin used (e.g., $N$-(deoxyguanosin-8-yl)-2-aminoflourene (dG-C8$\mathrm{AF})$ repair does not show strand specificity, although these lesions are thought to be processed by the same pathway as UV-photolesions), the organism and developmental stage (e.g., Drosophila embryonic cells show neither strand-specificity nor preferential repair) and the dose of genotoxin (e.g., preferential repair of CPDs is dose-dependent over the range of approx. $10-30 \mathrm{~J} / \mathrm{m}^{2}$ : higher doses are believed to abolish preferential repair in active genes through prolonged inhibition of both transcription initiation and elongation). Some questions for the future include determining the effect on repair of acetylation/methylation and the relationship of the gene under study to the nuclear matrix.

P. Cooper (University of Berkeley, USA), addressed the topic of transcription-coupled repair of ionizing radiation induced DNA base damage in XP-G and CS human cells. It was previously shown that ionizing radiation can influence transcription initiation, that TCR of ionizing radiation induced DNA damage exists in human cells, and that the process was defective in cells from CS individuals. The present work extended these findings to the study of induction and repair of ionizing radiation induced thymine glycols (a form of oxidative damage) in cells from another NER defective condition, XP-G. Thymine glycols were detected by specific antibodies in an ELISA assay. XP-G is a rare XP complementation group, with individuals showing a diverse clinical spectrum. XP-G cells (the XP3BR fibroblast cell line) were first shown to be ionizing radiation sensitive in 1980 by C. Arlett: their sensitivity is approx. 2-fold compared with wild-type fibroblasts. This cell line was also found to have slightly higher sensitivity to high linear energy transfer (LET) radiation than wild-type. The ionizing radiation-sensitive XP-G cell lines were shown to harbour a defect in the transcription-coupled repair of thyminc glycols. The thymine glycol repair defect was found to be associated with some, but not all, XP-G cell lines. It was suggested that the XPG protein may have a function additional to its role in NER: its involvement in a possible process coupling transcription to oxidative damage repair.

Biophysical studies addressing the effects of chromatin on radiosensitivity were presented by $\mathbf{S}$. Chiu (Case Western University, USA) and E. Woudstra (Groningen, The Netherlands). The former speaker addressed DNA-protein crosslink (DPC) formation after ionizing radiation. Characteristics of these heterogeneous lesions include a yield of approx. $3 \%$ of the number of DNA single-strand breaks induced by low-LET radiation (one estimate is 34 DPCs/Gy/cell) and an increase in their production under hypoxia (factors of 1.4-2.3-fold have been found). Evidence was presented that histones are rarely involved in DPCs, with nuclear matrix proteins being the usual DPC protein substrate. It was suggested that the repair of DPCs may be by differ ent pathways than those used for DNA dsb repair. This suggestion was based on the fact that there was no detectable DPC repair defect in the $X R-1$ or $x r s 6$ mutants (XRCC4 and XRCC5 genes, respectively; Table 1) in comparison to wild-type. However, lack of involvement of other DNA dsb repair genes in DPC repair was not excluded in these studies. The latter speaker reported on studies which used mammalian cell lines of differing radiosensitivity and differences in the induction of DNA dsbs, but in which DNA dsb repair was equivalent. Such lines were suggested to be good candidates for harbouring differences in chromatin structure affecting radiosensitivity. In an assay based on chromatin unwinding ('halo' assay), the radiosensitive HX142 neuroblastoma cell line showed greater post-irradiation unwinding. Further studies identified the lack of two proteins (of 49.5 and $34.8 \mathrm{kDa}$ ) in the radiosensitive HX142 cell line. It was suggested that these proteins were candidate DNA-matrix attachment region 
(MAR) proteins, based on the hypothesis that cell lines with an unstable MAR cannot restrict DNA dsbs to one DNA loop-matrix unit, resulting in greater chromatin unwinding. Attempts were in progress to identify the proteins concerned.

N. Oleinick (Case Western Reserve University, USA) discussed the role of DNA organization in eukaryotic nuclei in relation to hypersensitivity of certain regions for damage. The nuclear matrix is responsible for the formation of chromosomal loops of $50-100 \mathrm{kbp}$. These loops are formed through anchorage of matrix-attachment DNA sequences to the nuclear matrix. DNA in these loops is associated with fewer histones as a result of high transcription activity, replication and some types of repair. This renders these loops hypersensitive to, for example, ionizing radiation. In vitro formation of these loopcontaining matrices is achieved by creating an environment with increasing ionic strengths to release histones and other proteins.

P. Cook (University of Oxford, UK) also presented data that subscribes the theory of specific, active foci in the DNA-matrix. Active polymerases involved in replication, transcription as well as DNA-repair are concentrated in about 2500 foci in the nucleus of HeLa cells. An interesting observation was that foci involved in DNA repair/replication are not used for transcription and vice versa.

\section{Molecular and genetic aspects of cancer devel- opment}

Using illustrative data mainly obtained from colon cancer cell lines, E. Stanbridge (University of California at Irvine, USA), presented an overview of some of the genetics and molecular mechanisms of carcinogenesis. According to the model previously proposed by Loeb, a 'mutator' genomic instability phenotype can occur as a result of dysfunction of genes involved in fundamental processes such as mismatch or excision repair, DNA replication or chromosomal segregation. Tumor progression can thereafter ensue as a result of mutations in a secondary level of genes (e.g., ras, p53). Studies such as those performed with colon cancer cell lines have supported this hypothesis: genomic instability, assessed at various microsatellite loci, was reversed by somatic cell fusion, transfer of the relevant whole chromosome containing the mismatch repair locus absent in the cell line, or expression of the relevant cDNA. Such results showed that the condition was recessive. An important result was that the malignant phenotype was still maintained, suggested to be not unexpected because of the multiple genes involved in tumorigenesis. Other studies with the SW480 colon cancer cell line used microcell-mediated human chromosome transfer to examine the influence of restoration of wild-type copies of genes which were mutated in that cell line, with the endpoint being the effect on tumorigenicity. Sequential transfer of chromosome 18 (containing the $D C C$ wildtype gene) and chromosome 5 (containing the $A P C$ wild-type gene) reduced tumorigenicity by approx. $50 \%$ and over $90 \%$, respectively, whilst transfer of chromosome 17 (containing wild-type p53) resulted in tumour regression by an apoptotic mechanism. Whilst other genes on these chromosomes could clearly be affecting the results, transfer of irrelevant chromosomes as controls had no effect on tumorigenicity, and the chromosome 17 result was confirmed by cDNA expression of wild-type p53. Other studies showed that expression of activated ras had no observable tumorigenic effect on normal human cells, but resulted in phenotypic progression in transformed cell lines.

H. de The (Hopital Saint Louis, Paris, France), overviewed some leukemo- and lymphoma-genesis mechanisms resulting from different functional classes of proteins derived from transforming genes located at translocation breakpoints. Molecular techniques have proved very powerful in dissecting the relevant mechanisms. Three broad categories could be discerned. The first involves insertion of a promoter which deregulates the expression of an oncogene. An example is the classical $t(8: 14)$ translocation, where the strong promoter from the immunoglobulin $\mathrm{H}$ or T-cell receptor $\alpha$ loci on chromosome 14 are translocated to $\mathrm{c}-\mathrm{Myc}$ on chromosome $8 \mathrm{q}$. The second category is the formation of a chimeric gene by fusion of (part of) one protein with another. Typically, the two proteins are involved in either signalling cascade pathways or are transcription factors, and the fusion protein acts in both pathways. An example is the BCR-ABL translocation. A third mechanism involves fusion between a 
kinase domain and a nuclear protein, resulting in inappropriate protein phosphorylation within the nucleus. An example is the ALK-NPM fusion in nonHodgkins lymphoma. Medical uses of studying these proteins include the diagnosis and assessment of minimal residual disease (using RT-PCR) after chemotherapy or radiotherapy for leukemias and lymphomas, and the potential for developing tumour-specific antibodies.

Genetic disorders conferring specific risk for cancer development, especially after ionizing radiation exposure, were addressed by R. Cox (Didcot, UK). Second malignant (and occasionally benign) neoplasms occur at very low rates after therapeutic radiation for cancer at most sites. However, in some situations, the risk is greater. In sporadic retinoblastoma cases, the risk of later developing osteosarcoma within the irradiated field is approx. 2-fold increased, soft tissue sarcoma $>2$-fold increased and there is an increased risk of tumors of the brain. meninges and melanoma. This is presumably the result of the germline inactivation of one $\mathrm{Rb}$ allele in these individuals, and radiation-induced inactivation of the second allele. Individuals with Gorlin's (basal cell nevus) syndrome have a hugely increased risk of ionizing radiation-induced cancer, first noted when patients received radiation therapy for a rare type of cerebellar tumor to which they are predisposed (medulloblastoma). The sensitivity to tumour induction is in the form of multiple (often thousands) of basal cell carcinomas (and to a lesser degree, squamous cell carcinomas) on the irradiated skin, and an increase in pre-pubertal ovarian fibromas and fibrosarcomas in females. A point of note regarding the ovarian tumors appears to be a greatly reduced latency between irradiation and tumor formation, implying that the involved gene is probably a lumour suppressor gene. This speaker also indicated that in normal individuals, the breast is an organ with a particular increased post-irradiation tumour formation risk. For example, a 13.5-fold increase in premenopausal breast cancer has been reported in $\mathrm{A}$ bomb survivors. This is topical in view of previous suggestions that AT heterozygotes may be at increased tumor risk from screening mammography, a testable hypothesis now the ATM gene is cloned. The effect of p53 on ionizing radiation carcinogenesis was also addressed in this presentation. Previous work showed that p53 homozygous null mice are greatly predisposed to spontaneous tumor formation. After ionizing radiation, no increased tumor risk was seen in the homozygous null animals, presumably because of the high background cancer rate. Mice heterozygous for p53, analogous to the human condition, Li-Fraumeni syndrome (in which individuals harbour a germline inactivating mutation in one p53 allele) had a greatly increased post-ionizing radiation cancer incidence, with tumor levels intermediate between unirradiated or irradiated homozygous null animals and irradiated wild-type controls. Another point addressed by this speaker was the Eker rat: heterozygous rodents are at increased risk for ionizing radiation carcinogenesis and as such have proved a useful model system. The relevant gene had been localised to rat chromosome $10 \mathrm{q}$ by positional cloning: recently, the TSC2 (tuberous sclerosis 2) gene was cloned, revealing that it was the human equivalent of the Eker gene. As such, individuals with TSC2 mutations might also be expected to have enhanced tumorigenic radiosensitivity.

\section{Miscellaneous}

Regarding ionizing radiation mutagenesis, J. Thacker (Didcot, UK), identified three fundamental issues: the molecular spectrum of mutations, identifying which of these are important to carcinogenesis and hereditary diseases, and addressing what might happen to individuals with a compromised ability to process the mutations. Much current research is focussed on the first of these issues. This speaker stressed the importance of the systems used to examine mutations, as different systems (such as different species, different reporter genes) may give very different results. For example, the HPRT and APRT genes of hamster cells are commonly used for mutational analysis. Advantages of the former is its large size (and therefore suitability for analysing large mutations characteristic of ionizing radiation-induced mutations), and both genes are non-essential and in single copy. However, when post irradiation mutational spectra are analysed, there is a marked difference between the two genes, with the former showing many large mutations (deletions/rearrangements) and the latter a much greater incidence of 
point mutations. A possible explanation is that there might be an essential gene adjacent to APRT, deletion or rearrangement of which would destroy the APRT reporter function. The latter has experimental support in that in systems with multiple APR'T copies. many more mutants (and mutants with larger mutations) are recovered. In terms of the more common large deletions/rearrangements seen after ionizing radiation exposure, recent work from the speaker's laboratory has shown that when regions flanking such mutations were sequenced, direct DNA repeats were commonly found on either side. There has also been the recognition that ionizing radiation, as opposed to other mutagens, causes a greater incidence of so-called 'unconventional' mutational events, such as delayed or co-incident mutations or hereditable genetic instability. Possible explanations suggested for these data included alterations in chromatin. mobilization of transposable elements, persistent lesions or induction of an altered metabolic state (altered gene expression). Of topical interest was that AT shows, in general, a spontaneous increase in such unconventional mutational events.

G. McKenna (University of Pennsylvania, USA) reviewed the normal controls of the eukaryotic cell cycle before addressing the association between ionizing radiation and the cell cycle. Some notable themes have cmerged in the latter area over the last few years. One prominent theme is that of cell cycle checkpoints, feedback controls initially described in $S$. cerevisiae in response to ionizing radiation, which halt cell cycle damage in response to DNA damage, presumably to allow time for repair. Determining the mechanisms whereby the presumed signal arising from damaged DNA is transduced to the cell cycle regulators is of major interest. Whilst most is known about yeast checkpoints, another recent finding is that many of the genes regulating the yeast cell cycle and involved in checkpoint controls are conserved in mammals. A number of proteins critical to the cellular response to ionizing radiation damage also regulate the cell cycle checkpoints. Notable examples are ATM, p53 and p16/CIP1. Determining the exact roles such proteins play in cell cycle regulation and how they interact with the intrinsic cell cycle machinery is an area of intense recent interest.

R. Haynes (York University, Toronto, Canada) reviewed the history of the field of DNA repair. This report cannot do justice to this seminal lecture, but only touch on a few of the major points raised. The concept of DNA repair originated in the field of radiobiology/photobiology: the present meeting was held precisely 30 years after the first DNA repair conference, in the University of Chicago, 1965, one year after Setlow and Carrier and Boyce and Howard-Flanders independently described excision repair of pyrimidine dimers. Interestingly, the majority of early radiation research was initially concentrated on cellular ionizing radiation repair. With the discovery of the pyrimidine dimer photolesion and the development of assays for its study, many workers moved to UV repair, an area which developed into the active field it is today. The comment was made that many of the molecular tools developed in the study of UV radiation and its effects are now being applied to the study of ionizing radiation damage and repair, with numerous recent advances (as also addressed by a number of other speakers at this congress). Studies in the latter half of the 1960s with $E$. coli by Witkin were stated to have been instrumental in developing the major new concepts that repair was linked to mutation, and that mutation was not a physical process (as held in early studies by workers as prominent as Max Delbrück) but a biochemical process. The description by Cleaver in 1968 that XP was a human condition resulting from defective DNA repair was a landmark in demonstrating the (potential) importance of the developing field to human health. Two other cellular repair functions of major evolutionary significance were identified as the proofreading function after DNA replication and mismatch repair, the latter perhaps being the major speciation barrier.

\section{Closing statements}

Molecular genetic approaches are now yielding many new genes involved in the cellular response to ionizing radiation damage. It is expected that the number of new genes reported will increase substantially before the next radiation research congress. The next phase will be to determine the biologic roles of the protein products of the genes, arguably a more difficult task. Potential contributions of such work to human health are great, however, and in- 
clude, as for the study of UV damage processing, a greater understanding of the mechanism of ionizing radiation-induced carcinogenesis. An additional and major aspect of the potential of molecular radiation biology research is for a contribution to the management of individuals with cancer. For example, if one or a few genes prove to be involved in the majority of individuals with enhanced radiotherapy reactions, this could open the way for pre-radiotherapy genetic screening, whilst a greater understanding of radiation response genes could translate to novel approaches to the treatment of cancer with ionizing radiation.

\section{Acknowledgements}

Thanks are extended to Roland Kanaar for comments on the manuscript. 